\title{
A Comparative Study of Concentration Techniques for Detection of Intestinal Parasitic Infections - to Evaluate the Prevalence And to Identify A Better Method of Concentration Technique At A Tribal Tertiary Care Hospital
}

\author{
B. M. Shanker Venkatesh ${ }^{1}$, S. Rajeshwar Rao ${ }^{2}$, N.Vivekanand ${ }^{3}$, B.M.Shanker \\ Venkatesh ${ }^{4}$, \\ ${ }^{I}$ Associate Professor, Of Microbiology, RIMS Adilabad,T.S. \\ ${ }^{2}$ Associate Professor, Of Microbiology, Osmania Medical College, Hyderabad \\ ${ }^{3}$ Associate Professor, Of Pathology, RIMS Adilabad,T.S.,
}

Flat No. B305 Sai Pragathi Pride Apts. S.S Nagar, Street No.08, Habsiguda, Hyderabad.

\begin{abstract}
Parasitic infection caused by helminths and protozoa are the major cause of human disease in most countries of tropical region. It is estimated that about 3.5 billion people are infected with intestinal parasites of whom 450 million are ill. The prevalence of intestinal parasitic infection varies not only geographically but also in different region in the same country.

The present study is undertaken to determine the rate of prevalence of intestinal parasitic infection among all age group of people in this tribal area and to determine the best concentration technique to identify maximum number of intestinal parasites.

Material \& Methods: A total of 836 stools samples were collected from patients with symptoms of parasitic infestation and the same were subjected to (1) Gross examination (2) Direct microscopic examination by using saline and iodine preparation and (3) modified Ziehl Neelson staining after fixing with methanol (4) different concentration technique viz. (a) Brine concentration flotation technique (b) Zinc sulphate flotation technique (c) Formal ether concentration sedimentation technique (d) Merthiolate iodine formalin sedimentation technique.

Results: Out of total 836 stools samples the detection rate by various methods was $286(34.2 \%)$ for ova and cysts of protozoa, coccidian and helminths. Compared to females (29.75\%), males (38\%) were more affected.

The direct smear saline mount could only detect $36 \%$ while the maximum detection $66 \%$ of intestinal parasite was after Formalin ether concentrated sedimentation technique.

Among the intestinal protozoa, Entamoeba histolytica (53.49\%) was the most common followed by Giardia cysts (8.04\%) . Coccidian parasites like cryptosporidium parvum and isospora beli were detected in the HIV infected patient only.

Ascaris lumbricoides (16.43\%) followed by ancylostoma duodenele (9.09\%) were the common helminthic infections.
\end{abstract}

Conclusion: Early and prompt diagnosis of intestinal parasitic infection is important as in addition to morbidity and mortality they contribute to malnutrition, growth retardation and diminished work capacity.

\section{Introduction}

Parasitic infections caused by intestinal helminths and protozoans account for significant burden of human disease load in developing countries. It is estimated that around 3.5 billion people harbour intestinal parasites and 450 millions are ill as a result of these infections ${ }^{1}$. Poor sanitation, scarcity of potable drinking water and low standard of personal hygiene contributes to rapid spread of the infection ${ }^{2}$. The prevalence of different parasitic disease depends upon environmental, social and economic factors ${ }^{3}$. In India, malnutrition, improper disposal of sewage and non availability of potable water supplies especially in rural and tribal areas are responsible for the high rate of intestinal parasitic infections. ${ }^{4}$. The WHO reported that Entamoeba histolytica causes approximately 50 million cases and 1 lakh deaths annually ${ }^{5}$. The number of people who are affected by Giardia lamblia, whip worm, round worm and hook worm in developing world has been estimated to be $200,500,700,800$ millions respectively ${ }^{6}$.

Due to low density of parasites in the faeces, direct wet mount method can miss parasites (ova, cysts and larvae) and the detection can be enhanced through using concentration techniques. The present study was conducted with an aim to determine the prevalence of intestinal parasitic infection in a tribal tertiary care hospital and to compare the stool concentration techniques for detection and identification of intestinal parasites. 


\section{Material And Methods}

The present study was conducted in the Department of Microbiology, Rajiv Gandhi Institute of Medical Sciences Adilabad for a period of one year. A total of 836 stool samples were collected from patient attending out patients Department and admitted in wards with symptoms of intestinal infestation like diarrhea, vomiting, abdominal pain and weight loss and they were included for the study.

\section{Specimen Collection}

Freshly voided stool samples were obtained from these patients in sterile screw capped wide mouthed disposable plastic containers. Care was taken not to include samples which were contaminated with urine and also from patients already on medication. The samples were then transported to the Microbiology Laboratory immediately.

\section{In The Laboratory}

The specimen was subjected to

(1) Macroscopic examination was done and the color, consistency nature presence of mucus and blood were noted and also observation was made for presence of adult helminthic worms with the help of hand lens.

(2) Microscopic examination was done using direct preparation of (saline and iodine wet mount) for detection of trophozoites and cysts of protozoa and ova of helminths.

Modified Ziehl Neelson technique was done after methanol fixation for the specimens suspected to have been taken from HIV Patients for detection of coccidian parasites like cryptosporidium and isospora.

\section{Concentration Techniques Performed}

(1). Brine concentration flotation technique : saturated solution of $\mathrm{NaCl}$ (brine solution) is prepared and a small amount of faeces is mixed with $2 \mathrm{ml}$ of brine solution in a bijou bottle. More brine solutions added till the brim of bijou bottle while stirring. Drops of brim solution are added to the surface of bottle without over spilling. A clean glass slide is placed over the solution surface and left for 30 minutes exactly. The slide is lifted in single hand motion and examined under Microscope.

(2). Zinc sulphate centrifugal floatation technique : $1 \mathrm{~g}$ of the stool specimen was emulsified in 10 parts of tap water and it was strained through a wire gauze. The filtrate was collected in a Wassermann tube and centrifuged at $2500 \mathrm{rpm}$. The supernatant was discarded and sediment was re- suspended in water. This step was repeated till the supernatant became clear. To the sediment, 3 to $4 \mathrm{ml}$ of 33\% Zinc Sulphate solution was added, it was mixed well and it was filled with $\mathrm{ZnSO}_{4}$ solution, up to about half an inch of the rim. Several loop full of the supernatant fluid was removed with a bacterial logical loop and they were observed for parasites.

(3). Formol - ether concentration sedimentation technique (Allen and Ridely modification) : $1 \mathrm{~g}$ of stool was emulsified in $7 \mathrm{ml}$ of $10 \%$ formol saline and it was kept for $10 \mathrm{~min}$ for fixation. It was then strained through a wire gauze. The filtrate was added to $3 \mathrm{ml}$ of ether and it was centrifuged at $3000 \mathrm{rpm}$ for 60 seconds and allowed to settle. The supernatant was removed and a wet mount was made of the deposit to look for parasites.

(4). Merthiolate -iodine formalin concentration (MIFC) method : The following solution were prepared weekly and stored in amber coloured bottles ready for use. Solution 'A' consisted of tincture metrhiolate 200 $\mathrm{ml}$, distilled water $200 \mathrm{ml}, 40 \%$ formaldehyde $25 \mathrm{ml}$ and glycerine $5 \mathrm{ml}$. Solution 'B' consisted of iodine crystals $5 \mathrm{gm}$, potassium iodide $10 \mathrm{gm}$ and distilled water $100 \mathrm{ml}$. For each stool specimen, two tubes are prepared - one containing $9.4 \mathrm{ml}$ of solution 'A' and other $0.6 \mathrm{ml}$ of solution ' $\mathrm{B}$ ', the contents were mixed together immediately before adding to approximately $1 \mathrm{gm}$ of faeces, after the stool specimen emulsified thoroughly. The tube was stoppered and allowed to stand overnight. The contents are mixed again and filtered through surgical gauze the next morning. Ethyl ether is added and tube is shaken vigorously. After keeping the tube for $1 \mathrm{~min}$, centrifugation is carried out at $1800 \mathrm{rpm}$ for $2 \mathrm{~min}$. Four zones are formed with this technique, the faecal plug is separated and the upper three zones decanted. The sediment is thoroughly mixed and a drop was placed on a slide covered with a coverslip, and examined.

\section{Results}

A total of 836 stool samples were examined, out of which 286 (34.2\%) samples were positive for intestinal parasitic infestation, as was observed by the different parasitic diagnostic methods.

Overall, the prevalence of parastic infections in males and females was $38 \%$ and $29.75 \%$ respectively (table-2). Children who were up to $0-5$ years of age $(60 \%)$ had the highest prevalence of the parasitic infestations(table-3) 
The most common intestinal protozoa cyst isolated was Entamoeba hystolytica $(53.49 \%)$ followed by Giardia cysts $(8.04 \%)$ and the helminthic eggs isolated was Ascaris lumbricoides eggs (16.43\%) followed by Hook worm eggs $(9.09 \%)$ (table-1)

Dual infections were seen in 45/286 patients. The most common dual infection was the infestation of the Entameba histolytica cysts with Ascaris eggs.

Table 1: Prevalence of parasitic infestation

\begin{tabular}{|l|l|l|}
\hline Parasite & Total no isolated & \% \\
\hline Entamoeba hystolytica trophozoites and cysts & 153 & 53.49 \\
\hline Giardia cysts & 23 & 8.04 \\
\hline Entamoeba coli & 8 & 2.79 \\
\hline Cryptosporidium & 6 & 2.09 \\
\hline Isospora & 3 & 1.04 \\
\hline Ascarislumbricoides eggs & 47 & 16.43 \\
\hline Hookworm eggs & 26 & 9.09 \\
\hline Hymenolepis nana eggs & 7 & 2.44 \\
\hline Trichuris trichura eggs & 6 & 2.09 \\
\hline Enterobius vermiculars & 7 & 2.44 \\
\hline TOTAL & $\mathbf{2 8 6}$ & \\
\hline
\end{tabular}

Table 2: Showing Sex prevalence of parasitic infection

\begin{tabular}{|l|l|l|l|}
\hline Sex & No of cases & Positive & \% \\
\hline Male & 436 & 167 & $38 \%$ \\
\hline Female & 400 & 119 & $29.75 \%$ \\
\hline
\end{tabular}

Table 3: Showing age wise prevalence of parasitic infections

\begin{tabular}{|l|l|l|l|}
\hline Age & No of cases & Positive & \% \\
\hline $0-5 y r s$ & 178 & 107 & 60 \\
\hline $6-10 y r s$ & 39 & 17 & 43 \\
\hline $11-20 y r s$ & 161 & 59 & 36 \\
\hline $21-30 y r s$ & 284 & 68 & 24 \\
\hline $31-40 y r s$ & 153 & 29 & 19 \\
\hline $40 a n d$ above & 21 & 6 & 28 \\
\hline Total & 836 & 286 & 34 \\
\hline
\end{tabular}

Table 4: Sensitivity of different parasitic examination methods

\begin{tabular}{|l|l|l|}
\hline Procedure & $\begin{array}{l}\text { No positive for parasites } \\
(\mathbf{2 8 6})\end{array}$ & $\mathbf{\%}$ \\
\hline Direct smear (Saline and iodine mount) & 102 & 36 \\
\hline Brine concentration flotation technique & 125 & 44 \\
\hline Zinc sulphate centrifugal floatation & 157 & 56 \\
\hline $\begin{array}{l}\text { Formol - ether concentration sedimentation(allen and } \\
\text { Ridely modification ) }\end{array}$ & 188 & 66 \\
\hline $\begin{array}{l}\text { Methiolate-Iodine Formalin sedimentation Concentration } \\
\text { (MIFC) method }\end{array}$ & 183 & 64 \\
\hline
\end{tabular}

The usually followed diagnostic method in the laboratory is the saline/ Iodine wet mount which could demonstrate only poorly with a sensitivity of $36 \%$ (102/286).

In this study the most sensitive concenterated method was found to be the Formol - ether concentration sedimentation(allen and Ridely modification) with a sensitivity of $66 \%$ (188/286 ) (table-4)

\section{Discussion}

Intestinal parasitic infections rank among the most significant causes of morbidity and mortality in the world ${ }^{7}$.In the present study the protozoan intestinal parasite Entamoeba histolytica followed by Giardia lamblia were the most prevalent species. This is in agreement to study made by Harsh Ahmed Amin and Shahnaz Abdul Khadar Ali ${ }^{8}$.This is also in agreement to study made by Parameshwarappa and Chandrakanth ${ }^{9}$ whose study also reported a prevalence of about $65 \%$ of Entamoeba histolytica among the isolates of intestinal parasite.Entamoeba histohytica is responsible for approximately 50 million cases of invasive amoebiasis and about more than 1 lakh deaths annually (WHO 1997). This may be due to their chlorine resistant status and its frequent contamination with food and water (Petri and Singh 1999). The next intestinal parasite of increased prevalance that was isolated was the helminth ascaris lumbri coides $(16.43 \%)$. This finding is comparable to the results of Parameshwarappa et al and Marothi Y. et $\mathrm{al}^{10}$ and Bishh D et al ${ }^{11}$ who also reported similar incidence in their study ${ }^{12}$. Several studies have demonstrated a high prevalence of intestinal helminthic infections in under- 
privileged community ${ }^{13}$. It is estimated that $25 \%$ of world population are infected with ascaris lumbricoides and this causes up to a million cases of deaths annually ${ }^{13}$. Intestinal helminthic infections are common in poor socioeconomical status in tropical and sub tropical region because of poverty,over crowding poor environmental sanitation and low level of education ${ }^{13}$.The frequency of parasitic infections differs with age and sex of general population. Intestinal parasitic infections are more common in children ${ }^{14}$. The prevalence rate of parasitic infection was higher in males $(38 \%)$ as compared to females $(29.75 \%)$. In a similar study by Parameshwarappa et $\mathrm{al}^{9}$ has reported a prevalence rate of $(33.39 \%)$ in males and $(21.29 \%)$ in females.

The reason for male preponderance in our study may be related to daily activity rather than sex preponderance. However sex predominance for parasitic infections is still not confirmed. Children under 5 years of age $(60 \%)$ has the highest prevalence of parasitic infection. This is an agreement to study conducted by Harsh Ahmed Amin et $\mathrm{al}^{8}$ who reported $(81 \%)$. Similar prevalence rates were reported by Al-Kubaisy et $\mathrm{al}^{15}$ and Farhan et $\mathrm{al}^{16}$ which showed the infection declined progressively with age.The prevalence in all age groups is high and it may be attributed to poor personnel hygiene and environmental exposure in this tribal area.

The diagnosis of parasitic infections in humans is challenging and it requires skill to identify. Routine diagnostic methods such as wet mount lack sensitivity. Concentration methods must therefore be adopted for increasing the sensitivity for identification of intestinal parasites in stool.

In the present study, a comparison has been made between different concentration techniques along with direct wet mount and it was found that there is a significant increase in the number of parasites after application of concentration techniques.

As per our study, formal ether concentration sedimentation (Allen \& Ridely modification) was more sensitive $(66 \%)$, followed by MIFC $(64 \%)$, Zinc Sulphate centrifugal flotation $(56 \%)$, brine concentration flotation technique $(44 \%)$ and the lowest detection was by wet mount technique (36\%). A similar study by Dr. Jai Shree Puri et $\mathrm{al}^{12}$ reported prevalence of intestinal parasites by formal ether concentration technique was $26.75 \%$ while it was $17.64 \%$ by Zinc Suplhate. A similar study by Moges F et al ${ }^{17}$ reported formal ether concentration technique as more sensitive compared to the other methods. Another study by Parameshwarappa et $\mathrm{al}^{9}$ reported formal ether method to be the most sensitive method with $64.5 \%$ detection.

However, in a study by Hersh Ahmed Amin et $\mathrm{al}^{8}$, the sensitivity of Zinc Suplhate flotation was found to be $49.3 \%$ while formal ethly sedimentation concentration yielded a sensitivity of $43.3 \%$.

As per our study, direct wet mount was less sensitive (36\%) when compared to concentration method. However, it had the advantage of being able to provide a quick diagnosis of a heavily infected specimen.

The concentration of stool allows detection of parasites even though in small numbers in stool where the direct smear fails to reveal any parasite. The present study showed that there is a significant increase in the number of parasites which were detected after the concentration techniques were applied.

Thus the formal ether concentration technique is recommended as it is easier to be perform, allows recovery of the broadest range of organisms and is least subject to technical error ${ }^{18 .}$ It must be included in stool examination detection of intestinal parasites, especially in rural and tribal setup as it is cost-effective and requires minimum basic infrastructure.

\section{Conclusion}

Intestinal parasites are world-wide in distribution and their prevalence in rural and tribal areas is high due to illiteracy, lack of personal hygiene, lack of access to potable water, poor sanitation. The climatic condition favor the development and survival of parasites and some of the factors contribute to high level of intestinal parasitic transmission.

Early and prompt diagnosis of intestinal parasitic infections is important as this contributes to malnutrition, growth retardation and diminished work capacity, in addition to morbidity and mortality. The formal ether sedimentation concentrated technique along with merthiolate iodine formalin sedimentation technique have high sensitivity. This can be supplemented by Zinc Suplhate flotation concentration technique and can be adopted in the laborataries to increase the diagnostic sensitivity.

\section{References}

[1]. M.Endris.Z Tekeste, W Lemma and A.Kassu, comparison of the Kato-Katz, wet mount and formal ether concentration diagnostic techniques for intestinal heliminth infections in Ethopia. ISRN parasitology, volume 2013, article ID 180439, 5 pages 2013.

[2]. Celikoz A, Guler N, Goztop AY;Prevalence of intestinal parasites in three socioecnomically different regions of Sivas, Turkey. J Health, population and Nutrition $2005 ; 23: 184-91$

[3]. Reader MM Infections \& Infestations of GIT ;Margulis and Burhennes Alimentary Tract Roentgenology 1994 ; 881-951

[4]. Malta H, Hally A, Gillman ; J. Cl. Microbiology ; 2000 Jan 38 (1) 133-37

[5]. Walsh J.A 1986 , Problems in recognition and diagnosis of Amoebiasis ; Estimation of Global magnitude of morbidity and mortality : Rev . Inf . Diseases 8 ;228-238- Cross Ref- Medline

[6]. Setihi S, Sehgal R, Malla N, Dudley NL; The changing trends of the intestinal parasitic infections in Chandigarh ; A hospital based study; Indian J Medical Microbiology $2000 ; 18$ (3) 106-9

[7]. Gilles HM , Hoffman PS ; Treatment of intestinal parasitic infections. A review of Nitrozoniade. Trends in Parasitology 2002 ; 18 : $75-79$ 
[8]. Harsh Ahmed Amin \& Shahnaz Abdul Khader Ali ; International Journal of Current Microbiology \& Applied Sciences : Vol. 4 No.(5) 2015 pgs 991-996

[9]. Parameshwarappa KD , Chandrakanth; The prevalence of intestinal parasitic infestation and the Evaluation of different Concenteration techniques of stool exam ; Journal of Clinical and Diagnostic Research :2012 Sept (suppl) Vol. 6 (7) : 1181-91

[10]. Marothi Y, Singh B ; The prevalence of intestinal parasites in Ujjain MP India :Afr. J . Microbiology . Res :2011:5(18) : 2711-14

[11]. Bish D , Verma A ,Bhardwaj HD ; Intestinal parasitic infestation among children in semi urban Indian population ; Tropical Parasitology $2011: 1(2)$ 104-7

[12]. Jaishree Puri , Nilekar SL ; Comparison of stool concenteration methods ; Instinal Wdian Journal of Basic and Applied Medical Research : Sept. 2014 Vol. 3 Issue 4 : pgs 158-162

[13]. Gupta P Bala , Deb M ; Prevalence of intestinal parasitic infections in HIV infected individuals ; Indian Journal of Medical Microbiology $2013: 31: 161-65$

[14]. Eatson A ; Intestinal worm impair child health in the Phillipines ; BMJ $1999: 318: 214$

[15]. AL Kubaisy ; Intestinal parasitic diarrhea among children in Baghdad : Tropical Biomedicine 2014:31(3): 499-506

[16]. Farhan A ; Prevalence of intestinal parasitic infestation in Al Ambar province Iraq : Journal of University of Ambar :6(1):1-4

[17]. Moges I ; Comparison of Formal acetone concenteration method with direct iodine method and Formal ether concenteration method for examination of stool parasites : Ethopia Journal of Health $2010: 24(2): 148-151$

[18]. Neimister R , Logan A ; Hemo- D as a substitute to Ethyl acetate in concenteration techniques Journal of clinical Microbiology $1987: 25: 425-426$ 